\title{
A Survey on Sentiment Analysis Approaches in e-Commerce
}

\author{
Thilageswari a/p Sinnasamy, Nilam Nur Amir Sjaif \\ Razak Faculty of Technology and Informatics \\ Universiti Teknologi Malaysia \\ Kuala Lumpur, Malaysia
}

\begin{abstract}
Sentiment analysis represents the process of judging customers' behavior expression and feeling as either positive, negative or neutral. Hence, a tangle of different approaches for sentiment analysis is being used, reflecting analysis of unstructured customers' reviews dataset to guide and generate insightful and helpful information. The aim of this paper is to highlight research design of sentiment analysis and choice of methodological by other researchers in E-Commerce customers' reviews to guide future development. This paper presents a study of sentiment analysis approaches, process challenges and trends to give researchers a review and survey in existing literature. Next, this study will discuss on feature extraction and classification method of sentiment analysis of customers' reviews to have an exhaustive view of their methods. The knowledge on challenges of sentiment analysis underpins to clarify future directions.
\end{abstract}

Keywords-Sentiment analysis; e-Commerce; feature extraction; classification; customers' reviews

\section{INTRODUCTION}

Since COVID-19 is a pandemic globally and causing companies to not be able to operate normally due to lockeddown with business operators to doing e-Commerce to survive [1]. e-Commerce is a safe way for consumers who make purchases for essentials and non-essentials goods and services online while staying home during the lock-down phase. ECommerce is made possible via different online platforms. Online platform is known as e-commerce or Electronic commerce which is online transaction business used in buying and selling products through internet [2] [3]. Examples of eCommerce are Shopee, Lazada, Zalora and eBay. These are world famous platforms selling goods, necessary products or services over the internet. However, people are doubtful of buying products from online platforms [4]. According to USA survey, there are $81 \%$ internet users who are buying products from online platform [5]. These customers express their feedback on their purchased items or services by writing reviews online at the comments section. Hence, reading other customers' feedback, comments or reviews is important to understand more about the products or services. Customers' reviews also known as Word of mouth (WOM) [3] [6] help other customers or clients to understand about the products, services and retailers. The more convincing the reviews are, the more confident the potential customers or clients will feel toward the products or services and be convinced to select and purchase them. Though customers' reviews are vital to effective customers' decision to make the right choices, the increasing number of reviews will require a potential customer to spend more time and effort to go through each review thus affecting the decision making process to be quite tedious as the potential customer has to read each review and analyze the product or service involved before making the final decision [1] [2]. Thus, to assist customers to improve making purchase decisions, many reviews analysis methods are employed to extract useful information for customers. Sentiment analysis helps to identify and analyze customers' or clients' sentiments in their text reviews to extract and present specific information necessary to make better purchase decisions on products or services in E-Commerce. This paper contributes survey analysis results by other researchers on sentiment analysis methods future development.

The paper is organized as follows: after this introduction, level of sentiment analysis, method for identification and basic requirement of sentiment analysis is discussed in Section 2 . Section 3 outlines sentiment analysis process presented with supporting examples. Section 4 reviews studies on related works from other researchers in different backgrounds and ECommerce customers' reviews using sentiment analysis. Section 5 discusses comparative analysis table of sentiment analysis with different methods in e-Commerce. Section 6 present discussion on comparative analysis table. Finally in Section 6, the paper ends with conclusions and acknowledgements.

\section{SEnTIMENT ANALSIS LEVEL}

Nowadays, the huge number of reviews requires efficient method for analyzing [4]. Customers and retailers reading thousands of reviews manually take plenty of time to classify the reviews in e-commerce using sentiment analysis method. The volume of reviews stored like mountain which requires some effective classifier to identify valuable information from text. Sentiment analysis or opinion mining is useful to extract customer's behavior by analyzing and exploring customer's reviews in E-commerce [7] [8] [9]. Customers express their emotions by writing subjective judgement about the products in E-commerce [40]. Sentiment analysis also helps to categorize the unstructured text as positive, negative and neutral whereby summarizes judgement by customers in order to understand other customer's expression and strength better about product and retailer [7] [10] [11]. Unstructured sentiments refer to detailed opinion by customer about the product [8]. Some information is explicit and others are implicit features. There are three levels of sentiment analysis: 
Document-level Sentiment Analysis (DSA), Sentence-level Sentiment Analysis (SSA) and Aspect-level Sentiment Analysis (ASA).

\section{A. Document-Level Sentiment Analysis (DSA)}

A document talk about negative or positive sentiment is called DSA. It is extracting sentiments from whole document [8] [12] [13] . The scenario has applied the sentiment analysis of air purifier based on coarse-grained reviews whereby the researcher presented neural network model to identify semantics of sentences classification [14].

\section{B. Sentence-Level Sentiment Analysis (SSA)}

SSA means sentiment expressed in sentences which decide whether negative or positive. Whereby it is simple sentiment analysis for extracting sentiments or customer's experiences from sentences [8] [12] [13]. At sentence level, the researchers present phrase recursive autoencoder (PRAE) model to identify sentiment in sentences for analysis of coarse-grained reviews [14]. However, according to [14] document and sentences level sentiment analysis unable to fine-grained features from the words.

\section{Aspect-Level Sentiment Analysis (ASA)}

ASA is opinion that classifies by identifying entities and their properties by classification and extraction [13] [15]. Whereby, it is interested on opinion words only from the reviews such as "Love the Amazon show", it is clearly mentioned using the word love [12]. The aspect 'love' from the text is important feature extraction phase that needed for sentiment analysis method. At aspect level classification, researcher presents hybrid model for the analysis of finegrained product's features [14]. It also expressed out sentiment polarity for further prediction process [16].

\section{SENTIMENT ANALYSIS PROCESS}

Fig. 1 shows process flow of sentiment analysis where by divided three main stages: product feature extraction, sentiment classification and ranking alternative products [2].

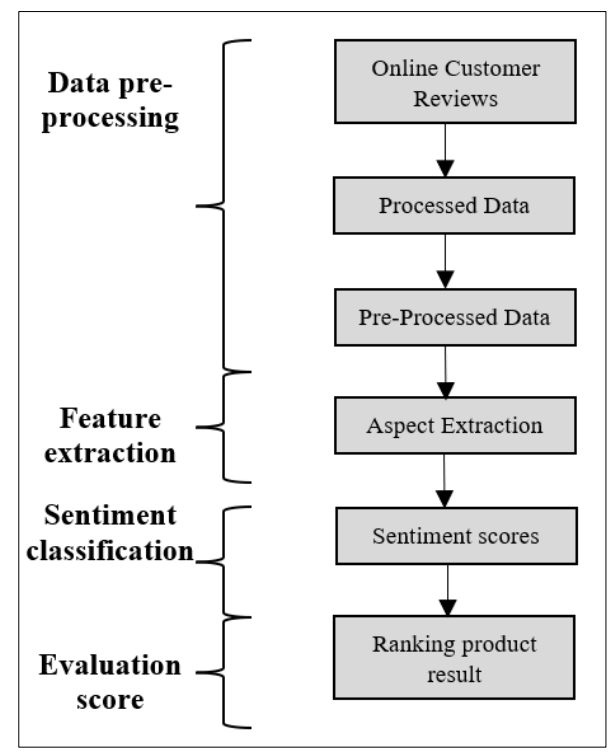

Fig. 1. Sentiment Analysis Process Flow [2] [4] [15].

\section{A. Preprocessed Texts}

As first step, data cleaning exhibit to clean unnecessary reviews from selected dataset [17] [18]. Data preprocessing perform to remove all missing values, remove stop words, tokenization, unwanted symbols, digits and URL tags [31]. Tokenization helps divide sentences into words, phrase or symbol and remove all stop words such as 'the', 'is', 'are' and ' $a$ ' [9]. The words required to convert to lower case as preparation for next step.

\section{B. Feature Extraction}

Aspect extraction from unstructured data helps extract all relevant information from dataset, reduces or removes irrelevant features of data for sentiment classification whereby the method is known as feature extraction [2] [19]. Feature extraction also helps extract implicit information from reviews other than explicit opinion to give more effective and better performance. There are few methods used for feature extraction :- frequent pattern mining with association rule mining, term document matrix (TDM), parts-of-speech (POS) tagging, Maximum entropy (ME), N-gram and lexicon [2] [8] [10] [16]. Those methods have advantages and disadvantages while applying for extract features in reviews. Frequent pattern mining is itemset, subsequence or substructures which helps find sequence database [20]. Apriori algorithm with association rules is one of the approach is in many fields. Other than that, according to U.A.Chauhan with other researchers has implemented Part-of-speech to find differences between noun, adjective, verb and adverb [5]. By extracting the term, in sentences reveals the hidden story and emotions of customers to be classified positive or negative. Furthermore, TDM is implemented to compute frequency of each word using method like bag of words and term frequency-inverse document frequency (TF-IDF) [5] [21] [22] [23]. TF-IDF helps to calculate number of times the word occurs and focuses on the importance term. By extracting most frequent words, researchers can ignore words with least scores. Some implement $\mathrm{N}$-gram features for extracting the features as unigram (One word), bigram (2 words) and trigram (3 words) whereby $\mathrm{N}$ represent number of words [22] [24]. Based on researchers, unigrams features commit to increase accuracy result in classification method. $\mathrm{N}$-grams helps to avoid semantic scores, the score calculation creates domain independent sentiment dictionary and computes to eliminate human annotators. These are some options by researchers for extract features from dataset before classifying the sentiment into positive, negative or neutral.

\section{Sentiment Classification}

Sentiment refers to feeling, emotions or responses of an individual by words for expressing human behavior and character [11] [25]. Hence, in this area explicit and implicit features that extract and identify hidden sentiment in measurable format. Whereby there are few methods to polarize the aspect in review theoretically: lexicon and machine learning classifier [2] [11] [13]. There are dictionary-based and corpus-based approaches for lexicon based such as Senti, HowNet, and Wordnet [8]. It is WorldNet dictionary which is stored with polarity positive, negative and neutral. Whereby automatically it is able to score the words in documents by 
counting number of positive and negative words in review [21]. If the review has more positive words than negative words, it is polarized as positive reviews. Some machine learning classifier for supervised learning are Naïve Bayes, Support vector machines (SVM), Maximum Entropy and Random forest [2] [9] [10] [26] [27] [31]. Fig. 2 shows summary overall sentiment classification based machine learning and lexicon approaches. Supervised learning required training labeled data to process output result based on input data [21], whereas unsupervised learning requires unlabeled training data to identify pattern of data output. Many researches used Naïve Bayes and SVM machine learning method for sentiment classification [15] [21].

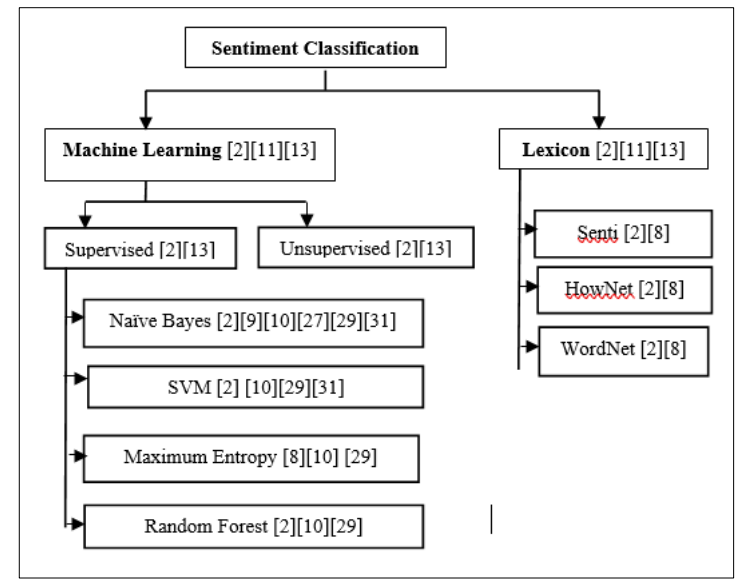

Fig. 2. Sentiment Classification Methods [2] [8] [9] [10] [11] [13] [26] [27] [31].

\section{Evaluation Score}

Based on feature extraction and sentiment classification on online reviews is rank the result using statical method [2]. The overall evaluation result is very important to judge subjective online reviews for customers. The result can predict or measure with mean squared error (MSA), confusion matrix, accuracy, precision, recall and F1-score [9] [15] [28] [29].

Equation of precision is presented as true positive (high quality reviews) divide by true positive (high positive reviews) + false positive (low quality reviews) [5] [18] [29].

Precision $=\frac{(\text { True } \text { Positive })}{\text { True Positive }+ \text { False Positive }}$

Recall represented as true positive (high quality reviews) divide by true positive (high quality reviews) + False negative (low quality reviews) [5] [18] [29].

Recall $=\frac{\text { True Positive }}{\text { True Positive }+ \text { False Negative }}$

F-score is calculated based on recall and precision as) [5] [18] [29]:-

$F_{\text {Score }}=\frac{2 * \text { Precision } * \text { Recall }}{\text { Precision }+ \text { Recall }}$

Mean absolute error (MAE) and Root Mean Square Error (RMSE) measure the closeness between fitted line to the data points [18] [29] [30].

$\mathrm{MAE}=\frac{1}{n} \sum_{1}^{n}\left|d_{i}-d_{i}^{\wedge}\right|$
Confusion matrix helps to show data difference between two classes [29]. Here, example of confusion matrix sketch with values True positive (TP), False positive (FP), False negative (FN) and True Negative (TN) as refer Fig. 3 [9] [15] [31].

TP: The output where correctly predicts the positive class.

FN: The output where incorrectly predicts the negative class whereby mislabeled as negative.

FP: The output incorrectly predicts the positive class whereby mislabeled as positive.

TN: The output where correctly predicts the negative class.

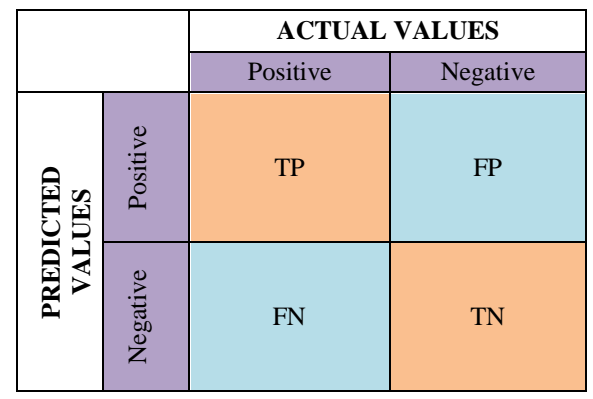

Fig. 3. Confusion Matrix for Amazon Dataset [9] [15] [31].

\section{RELATED WORK}

This section presents related studies of sentiment analysis on customer's reviews which conducted by researchers on ECommerce and different background of studies.

\section{A. Previous Work from Different Background}

Sentiment analysis is applied in different background of study. In Indonesia, Twitter status analyze with sentiment analysis method using SentiWordNet [32]. The emotion in tweet describes sentiment of user in Indonesian language whereby each sentences can polarize to positive, negative and neutral. Researcher used SentiWordNet which contains set of words score between 0 (negative) to 1 (positive). After scoring of sentiment, final result identified by sentiment classification method by calculating accuracy positive score 0.77 , neutral score 0.60 , negative score 0.78 and average score is 0.74 . Hence, emotions that express in words transform to meaning information. Other than that, sentiment analysis is also applied to extract Arabic opinions from text which is collected from twitter posts. In this research, Machine learning (ML) and Lexicon based (LB) approach with respect sentiment orientation is applied. The data collected and query using Tweepy Application Programming Interface (API) whereby positive and negative tweets are selected. N-gram features applied to divide the letter as Unigram (one word), bigram (two words) or trigram (three words). ML classifiers such as Naïve Bayes (NB), BNB, Multinomial NB (MNB), Maximum Entropy (ME), Support Vector Machine (SVM), Logistic Regression (LR), Stochastic Gradient Decent (SGD), RR, Adaptive Boosting (Ada-boost) and PA. To evaluate the performance of classifier accuracy, precision, recall and Fscore is used. Based on final result, single fold SVM 99.31 with unigram feature shows highest accuracy and 10 fold PA 99.96\% with unigram feature shows highest accuracy. The 
classifier helps to extract and discover the polarity of the given tweet. On other hand, movies' reviews and score in Rotten Tomatoes website predict with sentiment analysis method [33]. In this research, lexical based approach and supervised machine learning approach were used to predict sentiment polarity in movies' reviews. This researcher also has polarized the sentiment in review using SentiWordNet whereby it classified into two classes rotten (negative) and fresh (positive). The result evaluate by comparing proposed method and baseline method by calculating average precision, recall and $\mathrm{F}$ measure whereby proposed method shows highest result 0.97 . Hence, it is able to show better judgement on movies' reviews from rotten tomatoes website.

\section{B. Theory of Sentiments Analysis in E-Commerce}

This section discussed previous work related sentiment analysis in customers' reviews e-commerce. Amazon dataset on product's reviews has been selected by few writers for sentiment analysis. Sentiment analysis of unstructured data in Amazon dataset helps to measure and evaluate information in sentiment in reviews using natural language processing techniques [4] [7] [34]. Sentiment analysis was implemented for analysis of e-commerce product reviews to categorize negative and positive comments and visualize it in charts [4]. The model is developed with unigram and bigram and evaluate with classifier such as linear support vector machine,
Multinomial Naïve Bayes, Stochastic Gradient, Random Forest, Logistic regression and Decision tree by product category cellphone, musical and electronics [4] [27]. The result measure using accuracy, precision, recall and F-measure whereby linear support machine shows highest accuracy 93.57 better results compared to other papers. Text mining techniques Apriori and Term Frequency-Inverse Document Frequency (TF-IDF) were applied for identifying text features in proper way [23] [34].

\section{COMPARATIVE ANALYSis}

Table I shows implementation of sentiment analysis on review by some researchers based on Amazon dataset. The table presents many research and different sentiment analysis approaches toward resolving problems in e-commerce customers' reviews. Process of identifying sentiment from unstructured dataset provides different results as different methods are applied. The challenging part of sentiment analysis is to discover what customers like and dislike as written expression [15] [27]. The researchers used sentiment analysis method for identifying sentiment scores in online reviews and overall result as presented in Table I. Different researchers have conducted different approaches for feature extraction and sentiment classification, hence, some future improvement in method application is needed to attain greater accuracy.

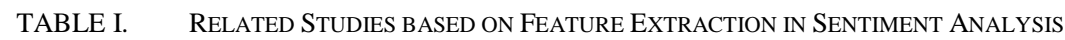

\begin{tabular}{|c|c|c|c|c|c|}
\hline Paper Title & Year & Dataset & Feature Extraction & $\begin{array}{l}\text { Sentiment } \\
\text { classification }\end{array}$ & Result \\
\hline \multirow{2}{*}{$\begin{array}{l}\text { Amazon Reviews business analytics with } \\
\text { sentiment analysis [35] }\end{array}$} & \multirow{2}{*}{2016} & \multirow{2}{*}{$\begin{array}{l}\text { Review of } \\
\text { Cellphone \& accessories }\end{array}$} & \multirow{2}{*}{ N/A } & MNB & Accuracy:- $72.95 \%$ \\
\hline & & & & SVM & Accuracy:- $80.11 \%$ \\
\hline \multirow{3}{*}{$\begin{array}{l}\text { Comparison of classification techniques } \\
\text { for Feature } \\
\text { Oriented Sentiment Analysis of Product } \\
\text { Review Data [27] }\end{array}$} & \multirow{3}{*}{2016} & \multirow{3}{*}{$\begin{array}{l}\text { Amazon fine foods:-214 reviews } \\
\text { [36] }\end{array}$} & \multirow{3}{*}{$\begin{array}{l}\text { POS tagging, } \\
\text { Bigram }\end{array}$} & Naïve Bayes & $\begin{array}{l}\text { Accuracy:- } 74.76 \% \\
\text { Precision:- } 79.54 \% \\
\text { Recall:-75.89\% } \\
\text { F-Measure:- } 73.75 \%\end{array}$ \\
\hline & & & & SVM & $\begin{array}{l}\text { Accuracy:- } 82.85 \% \\
\text { Precision:- } 84.45 \% \\
\text { Recall:- } 82.13 \% \\
\text { F-Measure:- } 82.38 \% \\
\end{array}$ \\
\hline & & & & $\begin{array}{l}\text { Maximum } \\
\text { Entropy }\end{array}$ & $\begin{array}{l}\text { Accuracy:- } 79.04 \% \\
\text { Precision:- } 81.75 \% \\
\text { Recall:-79.99\% } \\
\text { F-Measure:- } 78.59 \% \\
\end{array}$ \\
\hline \multirow{3}{*}{$\begin{array}{l}\text { Sentiment Analysis on Large Scale } \\
\text { Amazon Product Reviews [4] }\end{array}$} & \multirow{3}{*}{2018} & $\begin{array}{l}\text { Review of cellphone \& } \\
\text { accessories :- } 21600 \text { reviews [37] }\end{array}$ & \multirow{3}{*}{ TF-IDF } & \multirow{3}{*}{$\begin{array}{l}\text { Linear support } \\
\text { vector machine }\end{array}$} & Accuracy:- 93.57\% \\
\hline & & $\begin{array}{l}\text { Review of electronics:- } 24352 \\
\text { reviews [37] }\end{array}$ & & & Accuracy:- $93.52 \%$ \\
\hline & & $\begin{array}{l}\text { Reviews of music instruments :- } \\
2548 \text { reviews [37] }\end{array}$ & & & Accuracy:- $94.02 \%$ \\
\hline \multirow{2}{*}{$\begin{array}{l}\text { Sentiment classification on Amazon } \\
\text { reviews using machine learning } \\
\text { approaches [21] }\end{array}$} & \multirow{2}{*}{2018} & \multirow{2}{*}{$\begin{array}{l}\text { Amazon dataset :- } 252000 \\
\text { reviews from snap dataset }\end{array}$} & \multirow{2}{*}{ Bag of words model } & Naïve Bayes & Accuracy:- $92.72 \%$ \\
\hline & & & & SVM & Accuracy:- $93.20 \%$ \\
\hline $\begin{array}{l}\text { Classification of Amazon Book Reviews } \\
\text { Based on Sentiment Analysis [23] }\end{array}$ & 2018 & Amazon Book reviews [38] & TF-IDF & Random Forest & Accuracy:- $90.15 \%$ \\
\hline \multirow{2}{*}{$\begin{array}{l}\text { Aspect-Level Sentiment Analysis on E- } \\
\text { Commerce Data [13] }\end{array}$} & \multirow{2}{*}{2018} & \multirow{2}{*}{$\begin{array}{l}\text { Customer reviews from Amazon } \\
\text { products }\end{array}$} & \multirow{2}{*}{ Apriori algorithm } & Naïve Bayes & Accuracy:- $90.423 \%$ \\
\hline & & & & SVM & Accuracy :- $83.423 \%$ \\
\hline $\begin{array}{l}\text { Machine learning based aspect level } \\
\text { sentiment analysis for Amazon products } \\
{[15]}\end{array}$ & 2020 & Amazon Products :- [39] & N/A & $\begin{array}{l}\text { SVM classifier } \\
\text { (RBF Kernel, } \\
\text { BP-ALSA) }\end{array}$ & Accuracy:- 97\% \\
\hline $\begin{array}{l}\text { A comprehensive analysis of adverb types } \\
\text { for mining user sentiments on amazon } \\
\text { product reviews [5] }\end{array}$ & 2020 & $\begin{array}{l}\text { Amazon DVD musical product :- } \\
\text {.Net Crawler, } 9555 \text { reviews }\end{array}$ & $\begin{array}{l}\text { POS Tagging (Constituent } \\
\text { Likelihood Automatic Word- } \\
\text { tagging System (CLAWS)) }\end{array}$ & $\begin{array}{l}\text { Hybrid } \\
\text { approaches }\end{array}$ & $\begin{array}{l}\text { Precision :- } 0.89, \\
\text { Recall:- } 0.84, \\
\text { F-Measure:-0.86 }\end{array}$ \\
\hline
\end{tabular}




\section{DISCUSSION}

The survey table presents comparison result for years from 2016 till 2020 in sentiment analysis E-Commerce from various categories of Amazon dataset. Based on Table I, most of the method in sentiment analysis implement feature extraction and sentiment classification in their process flow for get better accuracy results. Many researchers have implemented text mining method for extract most frequent information from dataset like TF-IDF and Apriori algorithm. Machine learning supervised method is used in most of the papers for classification of information. The experimental results from frequent pattern mining and supervised machine learning methods are able to provide more than $90 \%$ accuracy result. For future investigation, lexicon method and result analysis needed for compare accuracy result with machine learning method in sentiment analysis of customers' reviews.

\section{VII.CONCLUSION}

In this paper, we have presented methodology of sentiment analysis and approaches based on previous studies in Ecommerce. Research studies results are to address customer satisfaction on online shopping platform based on other's reviews. Data analysis approach presents statical result for predicting and building strong confidence among customers who purchase product from online. Most researches have looked into many approaches and challenges, toward judging customers' behavior as discussed in different methods. The approaches are further applied in other field like Airline, Tourism, Hotel industry, hospitality and others. Sentiment analysis methodology and interpretation using analytic tools perform accurate result to customers. Hence, there are many challenges and ongoing more research in this area have to be discussed and improved to produce more efficient and reliable sentiment analysis approaches.

\section{ACKNOWLEDGMENT}

The authors would like to thank Ministry of Higher Education (MOHE) and Universiti Teknologi Malaysia (UTM) for their educational and financial support. This work is conducted at Razak Faculty of Technology and Informatics, under Cyber Physical Systems Research Group and funded by MOHE (FRGS: R.K130000.7856.5F026) and (UTM GUP: Q.K130000.2538.18H42).

\section{REFERENCES}

[1] L. T. T. Tran, "Managing the effectiveness of e-commerce platforms in a pandemic Lobel," sciencedirect, pp. 0969-6989, 2021.

[2] F. P. Zhi, G. M. Li and Y. Liu, "Processes and methods of information fusion for ranking products based on online reviews: An overview," Science Direct, pp. 87-97, 2020.

[3] S. Sanyala and M. Wamique Hisamb, "Factors Affecting Customer Satisfaction with Ecommerce Websites - An Omani Perspective," IEEEE, pp. 232-236, 2019.

[4] T. Ul Haque, N. Nawal Saber and F. Muhammad Shah, "Sentiment Analysis on Large Scale Amazon Product Reviews," IEEE, pp. 1-7, 2018.

[5] U. A. Chauhan, M. T. Afzal, A. Shahid, M. Abdar, M. E. Basiri and X. Zhou, "A comprehensive analysis of adverb types for mining user sentiments on amazon product reviews," Springer, p. 1811-1829, 2020.
[6] S. Al-Natour and O. Turetken Ted, "A comparative assessment of sentiment analysis and star ratings for consumer reviews," ScienceDirect, pp. 0268-4012, 2020.

[7] A. S. Rathor, A. Agarwal and P. Dimri, "Comparative Study of Machine Learning Approaches for Amazon Reviews," ScienceDirect, pp. 1552$1561,2018$.

[8] D. M. El-Din Mohamed and Hussein, "A survey on sentiment analysis challenges," ScienceDirect, pp. 330-338, 2018.

[9] P. Kencana Sari, A. Alamsyah and S. Wibowo, "Measuring eCommerce service quality from online customer review using sentiment analysis," IOP Publishing, pp. 1-7, 2018.

[10] T. Quyyam and D. H. Ghous, "Sentiment Analysis of Amazon Customer Product Reviews: A Review," ijsred, pp. 564-595, 2021.

[11] A. Ligthart, C. Catal and B. Tekinerdogan, "Systematic reviews in sentiment analysis: a tertiary study," Springer, pp. 1-57, 2021.

[12] M. Tubishat, N. Idris and M. Abushariah, "Explicit aspects extraction in sentiment analysis using optimal rules combination," ScienceDirect, pp. 448-480, 2021.

[13] M. B. Satuluri Vanaja, "Aspect-Level Sentiment Analysis on ECommerce Data," IEEE, pp. 1275-1279, 2018.

[14] Jing Zhang, Aijia Zhang, Dian Liu and Yiwen Bian, "Customer preferences extraction for air purifiers based on fine-grained sentiment analysis ofonline reviews," ScienceDirect, pp. 1-15, 2021.

[15] N. Nandal, R. Tanwar and J. Pruthi, "Machine learning based aspect level sentiment analysis for Amazon products," Springer, p. 601-607, 2020.

[16] M. Ahmed, Qun Chen and Zhanhuai Li, "Constructing domaindependent sentiment dictionary for sentiment analysis," Springer, pp. 14719-14732, 2020.

[17] M. Aman Ullah, S. Maliha Marium, S. Ara Begum and N. Saha Dipa, "An algorithm and method for sentiment analysis using the text and emoticon," ScienceDirect, pp. 357-360, 2020.

[18] A. Angelpreethi and D. S. BrittoRameshKumar, "An Enhanced Architecture for Feature Based Opinion Mining from Product Reviews," IEEE, pp. 89-92, 2017.

[19] G. Anand babu and B. Srinivasu, "A Conceptual Based Approach in Text Mining: Techniques and Applications," IJITEE, pp. 2278-3075, 2019.

[20] Jiawei Han, Dong Xin, Hong Cheng and Xifeng Yan, "Frequent Pattern Mining: Current Status and Future Directions," ResearchGate, pp. 1-33, 2019.

[21] S. Paknejad, "Sentiment classification on Amazon reviews using machine learning approaches," KYH Computer Science and Communication, pp. 1-23, 2018.

[22] R. Ahuja, A. Chug, S. Kohli, S. Gupta and P. Ahuja, "The Impact of Features Extraction on the Sentiment Analysis," ScienceDirect, pp. 341$348,2019$.

[23] K. S. Srujan, S. S. Nikhil, H. Raghav Rao, K. Karthik, B. S. Harish and H. M. Keerthi Kumar, "Classification of Amazon Book Reviews Based on Sentiment Analysis," Springer, pp. 1-12, 2018.

[24] A. Dey, M. Jenamani and J. J.Thakkar, "Senti-N-Gram : An n -gram lexicon for sentiment analysis," ScienceDirect, pp. 92-105, 2018.

[25] K. Chakraborty and A. Alla Hassanien, "Sentiment Analysis on a Set of Movie Reviews Using Deep Learning Techniques," Science Direct, pp. $1-14,2019$.

[26] K. Ashok Kumar, C. Jagadeesh, P. Kshirsagar and S. M. Marve, "Sentiment Analysis of Amazon Product Reviews using Machine Learning," Reseach gate, pp. 5245-5254, 2020.

[27] C. Pujari, A. and N. P. Shetty, "Comparison of classification techniques for Feature Oriented Sentiment Analysis of Product Review Data," ResearchGate, pp. 1-9, 2016.

[28] X. Dou, "Online Purchase Behavior Prediction and Analysis Using Ensemble Learning," IEEE, pp. 532-536, 2020.

[29] S. Saumya, J. Prakash Singh, A. Mohammed Baabdullah, N. P. Rana and Y. k. Dwivedi, "Ranking Online Consumer Reviews," ResearchGate, pp. 1-39, 2019. 
[30] Jicheng Li and Xinyue Huang, "Target customer selection method based on data mining in big data environment," IEEE, pp. 286-289, 2017.

[31] D. Shailendra Narayan Singh and Twinkle Sarraf, "Sentiment Analysis of a Product based on User Reviews using Random Forests Algorithm," IEEE, pp. 112-116, 2020.

[32] N. Maulidiah Elfajr and R. Sarno, "Sentiment Analysis using Weighted Emoticons and SentiWordNet for Indonesian Language," IEEE, pp. 234238, 2018.

[33] Suhariyanto, Ari Firmanto and Riyanarto Sarno, "Prediction of Movie Sentiment based on Reviews and Score on Rotten Tomatoes using SentiWordnet," IEEE, pp. 202-206, 2018.

[34] A. L. Robert Ireland, "Application of data analytics for product design: Sentiment analysis of online product reviews," ScienceDirect, p. 128144, 2018.

[35] E. M. Soledad and Y.-F. W. , "Amazon Reviews, business analytics with sentiment analysis," ResearchGate, 2016.
[36] Krevl and J. Leskovec, "SNAP Datasets," June 2014. [Online]. Available: http://snap.stanford.edu/data.

[37] McAuley, Ruining He and Julian, "Ups and downs: Modeling the visual evolution of fashion trends with one- class collaborative filtering.," Feb 2016. [Online]. Available: https://arxiv.org/abs/1602.01585.

[38] ZhiLiu, "UCI:- Machine Learning Repository," 1106 2011. [Online]. Available:https://archive.ics.uci.edu/ml/datasets/Amazon+book+reviews

[39] R.Mitchell, "Web scraping with python: collecting more data from the modern web," April 2018. [Online]. Available: https://www.oreilly.com/library/view/web-scrapingwith/9781491985564/.

[40] A. Alamoodi, B. Zaidan, A. Zaidan, O. Albahri, K. Mohammed, R. Malik, E. Almahdi, M. Chyad, Z. Tareq, A. Albahri, H. Hameed and M. Alaa, "Sentiment analysis and its applications in fighting COVID-19 and infectious diseases: A systematic review," ScienceDirect, pp. 2-13, 2020. 